\title{
Méthode stroboscopique à éclairs retardés pour l'étude des bulles
}

\section{A delayed-flash stroboscope for bubble studies}

\author{
PAR M. BÉHAR,
}

INGÉNIEUT AU SERVICE DES TRANSFERTS THERMYQUES DU CENTRE D'ĚTUdES NUCLÉAIRES DE GHENOBLE

\begin{abstract}
L'appareil permet l'observation de phénomènes qui, sans être rigoureusement périodiques, sont suffisamment reproductibles.

L'éclair du stroboscope est déclenché par l'interruption d'un faisceau lumineux de commande après un retard réglable de $0 \dot{a} 100 \mathrm{~ms}$. On peut ainsi observer de facon continue la croissance, les interactions et la coalescence des balles.
\end{abstract}

\section{1. - INTRODUCTION}

On connaît l'intérêt du stroboscope dans l'étude des mouvements périodiques, mais son utilisation peut également s'étendre aux cas où la périodicité n'est plus rigoureusement assurée et où seule demeure, au cours de cycles successifs, une certaine reproductibilité de forme. Dans ces conditions, si l'on veut parfaitement immobiliser le phénomène, il est nécessaire que ce soit celui-ci qui commande le dẻclenchement de l'éclair du stroboscope. Alors se pose le problème de l'exploration des différentes phases de l'ćrolution du phénomène au cours du cycle; deux solutions sont possibles :

- choisir et faire varier la phase du mouvement qui commandera le déclenchement de l'éclair;

- fixer une phase particulière et introduire, entre le système de déclenchement et la lampe à éclairs, une ligne à retard variable.

\begin{abstract}
This apparatus enables phenomena to be ob. served which, though not strictly periodical, are nevertheless adequately reproducible.

The stroboscope flash is triggered off by the interruption of a luminous control beam after a time interval which can be set to any value up to 100 millisecondes. It thus provides a means of continuous observation of bubble growth, interaction and coalescence.
\end{abstract}

Cette méthode stroboscopique modifiée a été appliquée à l'étude de l'injection des gaz dans les liquides par bullage, ainsi qu'aux interactions entre colonnes de bulles injectées simultanément. Nous décrirons donc l'appareillage particulier utilisé dans cette étude, laquelle présente un certain intérêt en génie chimique ainsi que dans la simulation de l'ébullition.

\section{2. - DESCRIPTION SOMMAIRE DU PHENOMENE ETUDIE}

Lorsqu'à l'aide d'un orifice calibré, on injecte un gaz dans un liquide, par exemple de l'air dans de l'eau, on constate, que pour une certaine plage de pression d'injection, les bulles sont émises avec une assez grande régularité, leur fréquence moyenne (dépendant entre autres du diamètre de l'orifice et de la pression) se situant dans nos essais entre 10 et 100 bulles par seconde. C'est donc un phénomène a priori justi- 
ciable de la prise de vue cinématographique à l'ultra-ralenti, mais celle-ci entraîne de très grosses servitudes de moyens et de délais, que l'on a précisément cherché à éviter par l'emploi de cette extension de la méthode stroboscopique.

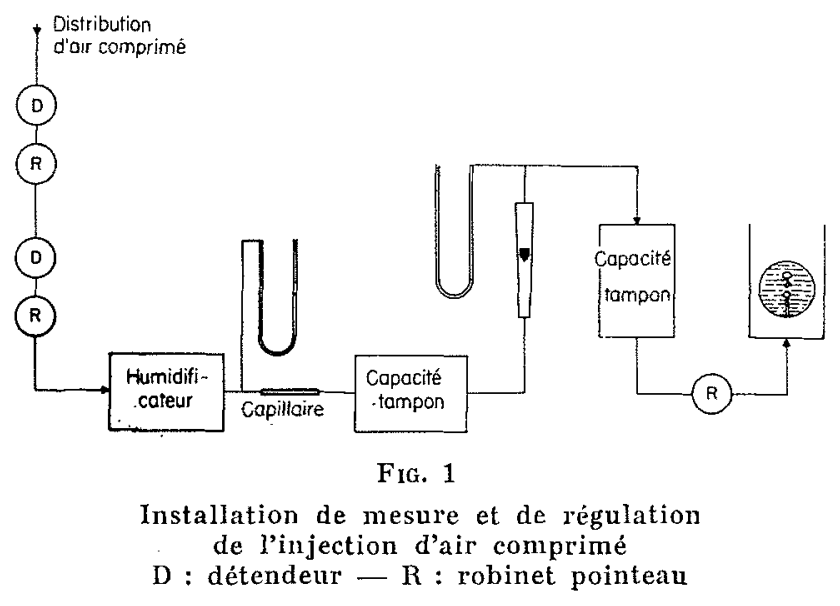

Les bulles successives passent, au cours de leur phase de croissance, exactement par les mêmes formes; de même, au début de leur ascension dans le liquide, les vibrations dont elles sont le siège leur donnent une même forme à une même hauteur au-dessus de l'injecteur; ensuite, évidemment, la dispersion des trajectoires rend impossible la stroboscopie. La coalescence de deux bulles, qui pourtant semble un phénomène très aléatoire, peut aussi, dans certaines conditions, s'observer par voie stroboscopique.

Pour obtenir une injection de bulles aussi régulière que possible, il est nécessaire de prendre certaines précautions : il faut qu'entre tubes d'injection et distribution de gaz comprimé (régularisé par mano-détendeur), se trouve un certain nombre de capacités-tampon, de tubes capillaires et de systèmes à perte de charge réglable (fig. 1). Il faut aussi que les appareils de mesure : micro-débitmètres à flotteur rotatif, manomètres à tubes en $U$, apportent le moins de perturbation possible (on peut observer, dans certains cas, des oscillations de couplage entre le liquide manométrique et l'injection des bulles). Tout ceci se justifie par le fait que le phénomène ne doit s'écarter que très peu d'une périodicité absolue, afin que la fatigue visuelle causée par l'irrégularité des éclairs soit réduite au minimum.

\section{3. - SYSTEME DE COMMANDE DES ECLAIRS}

Il suffit, dans notre cas, de faire couper par les bulles le faisceau lumineux d'excitation d'une cellule photo-électrique. Le stroboscope utilisé
(E.M.I. type 6) comporte une entrée spécialement adaptée à la cellule et il est encore possible de faire, dans une telle utilisation, la lecture de la frécuence moyenne. On pourra considérer la période de reproductibilité du phénomène comme s'étendant de la naissance de la bulle sur l'injecteur jusqu'à la fin de la partie régulière de sa trajectoire (soit environ quatre à cinq diamòtres de bulle au-dessus de l'orifice). Dans cet intervalle, il suffira de choisir l'intersection de la bulle et du faisceau lumineux de commande en réglant la hauteur de ce dernier au-dessus de l'injecteur.

Cette partie de l'appareillage comprend (fig. 2) :

- Une lampe à arc, suffisamment éloignéc du montage pour qu'elle soit vue de celui-ci comme une source ponctuelle;

- Un condenseur achromatique d'assez longue focale (en pratique au moins $100 \mathrm{~mm}$ ) afin d'obtenir un faisceau très délić. Ce condenseur devra former une image ponctuelle de la lampe à arc exactement à la verticale et au-dessus de l'injecteur, afin de localiser au mieux l'intersection de la bulle et du faisceau;

-- Un miroir de renvoi, situé dans le liquide, permettant de distribuer le faisceau lumineux vers l'injecteur avec lequel on dísire être synchronisé lorsque deux injecteurs fonctionnent simultanément;

--. Une cellule photo-électrique située au-dela des deux injecteurs. Il est à noter que l'on doit prendre certaines précautions afin que

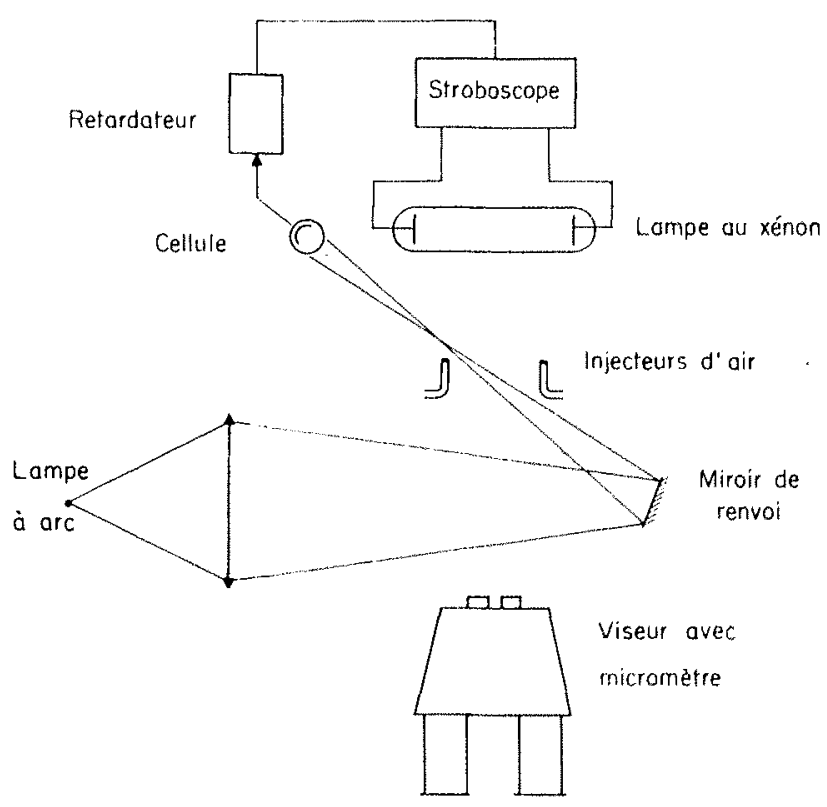

Fic, 2

Système de commande des éclairs stroboscopiques. 
la cellule ne soit influencée que par le faisceau lumineux de commande, bien qu'elle se trouve dans le champ lumineux de la lampe à éclairs; faute de quoi, cette dernière et la cellule formeraient un système auto-entretenu.

Chacun des organes précédents possède un nombre de degrés de liberté suffisant pour assurer au mieux les réglages. En particulier le condenseur a un déplacement vertical commandé par vis micrométrique.

\section{4. - SYSTEME RETARDATEUR D'ECLAIRS}

Des deux moyens indiqués dans l'introduction permettant l'exploration des différentes phases du mouvement, le second se montre, et de loin, le plus intéressant par sa souplesse d'utilisation et sa précision. Le retard entre la cellule photo-électrique et la lampe à éclairs est obtenu par un montage électronique (fig. 3) comprenant

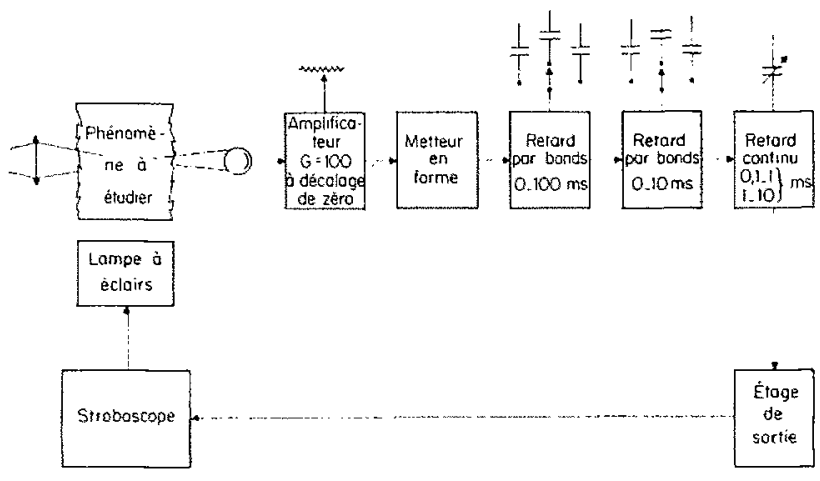

Fri. 3

Schéma d'ensemble du retardateur électronique.

des circuits monostables à transistors montés en série. Le schéma en est classique; il comporte notamment, à l'entrée, un amplificateur à sensibilité variable afin de tenir compte de différentes conditions d'éclairement de la cellule. Le temps de retard total, qui dépend un peu de la fréquence, atteint 100 millisecondes, ce qui permet de recouvrir largement une période et même de la dépasser, lorsque, par exemple, on veut suive un phénomène par continuité sans avoir à retourner en arrière. Le retard se fait par bonds de 10 millisecondes à l'intérieur desquels deux verniers, l'un par bonds de 1 milliseconde, l'autre continu, soit de 0 à 10 millisecondes, soit de 0 à 1 milliseconde, permettent l'ajustage du temps de retard. On se fixe arbitrairement le temps zéro par ajustage de la hauteur du faisceau lumineux au-dessus de l'injecteur. Comme il a été dit, il faut rendre la cellule insensible à l'allumage de la lampe à décharge lorsque le temps de retard n'est pas nul, car alors la lampe fournit des signaux parasites engendrant à leur tour des éclairs nuisibles à la bonne observation.

\section{5. - CONDITIONS D'OBSERVATION}

Le champ d'observation est tel que l'on peut y voir en même temps, suivant la fréquence, deux à trois images de bulles issues d'un même injecteur. On peut les observer soit à l'aide d'une loupe binoculaire munie d'un oculaire micrométrique, soit, la puissance de la lampe à décharge étant suffisante, projeter leur image sur un écran dépoli fait de papier translucide Kodatrace, ce qui permet de dessiner aisément leur forme. On peut donc changer certains paramètres du phénomène (par exemple la pression d'injection du gaz ou le diamètre de l'orifice) et enregistrer corrélativement leur influence, grâce aux dessins relevés, ce qui aurait exigé, en cinématographie ultra-rapide, la prise de vues sur de nombreux films.

A partir d'une fréquence de 12 à 15 bulles/ seconde et jusqu'à 20 ou 30 , la fatigue visuelle est légèrement supérieure à celle ressentie lors d'une projection cinématographique, car on a un seul éclair émis par période au lieu de trois, ce qui augmente le scintillement. De 5 à 10 bulles/ seconde, la persistance des impressions rétiniennes permet encore d'immobiliser le phénomène, mais alors la fatigue visuelle est très intense et on ne peut observer que pendant de courts instants.

II est à noter que lorsque deux injecteurs fonctionnent simultanément, la synchronisation n'étant assurée que pour un seul injecteur, l'aụtre donne en général une émission de bulles ayant, par stroboscopie, une certaine fréquence apparente. D'autre part, lorsqu'on étudie la coalescence, le faisceau lumineux de commande est fixé à la hauteur de la bulle commune et l'on s'aperçoit alors que dans certaines conditions, le phénomène est très reproductible, laissant voir notamment le déplacement de rides capillaires à la surface de la bulle et les déformations en volume de celle-ci (phot. 1 à 4 ).

En conclusion, il semble que dans le domaine de l'hydrodynamique en particulier, de nombreux cas de mouvements périodiques puissent être analysés par cette méthode.

Le montage n'a été réalisé dans sa forme définitive que grâce à de nombreuses suggestions de M. R. SÉméria du Service des Transferts Thermiques du Centre d'Etudes Nucléaires de Grenoble. La construction a été entièrement effectuée par MM. Chevrier et A. Gouzy du même Service; le système électronique retardateur est dû pour sa conception et sa réalisation à $M$. $H$. Chevalier du Service d'Electronique du Centre d'Etudes nucléaires de Grenoble. 


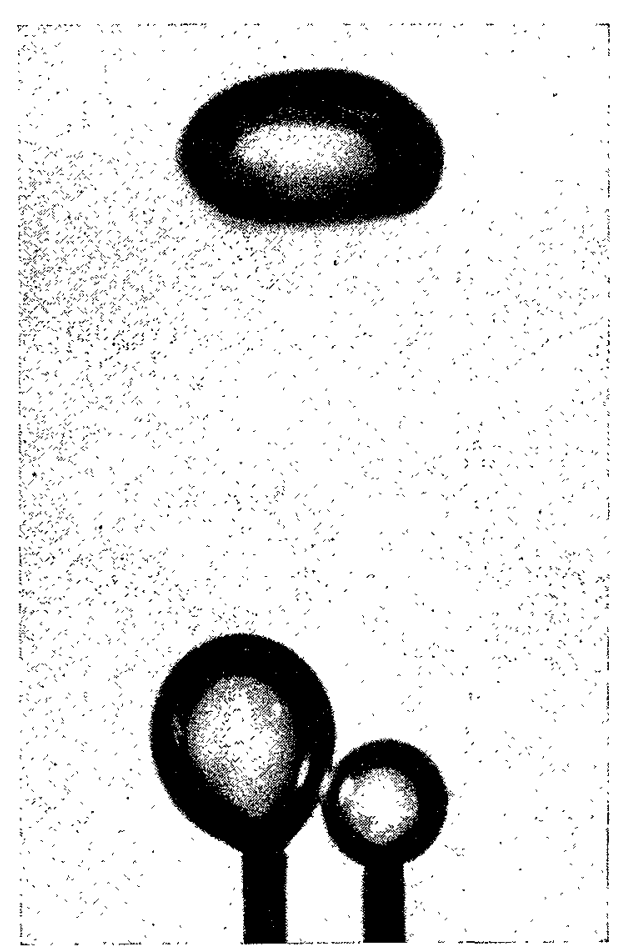

Pнот. 1

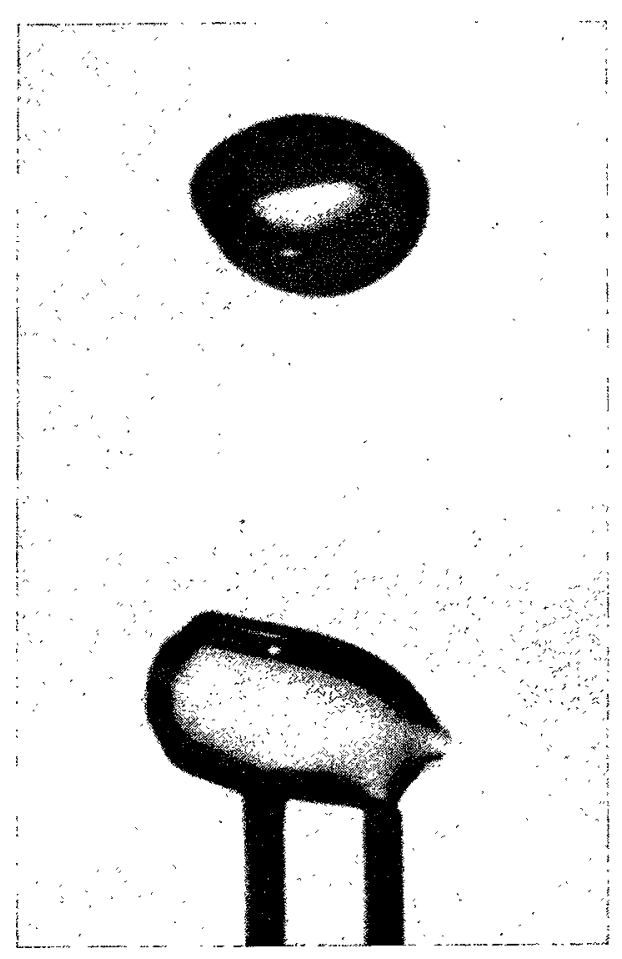

Pнот. 3

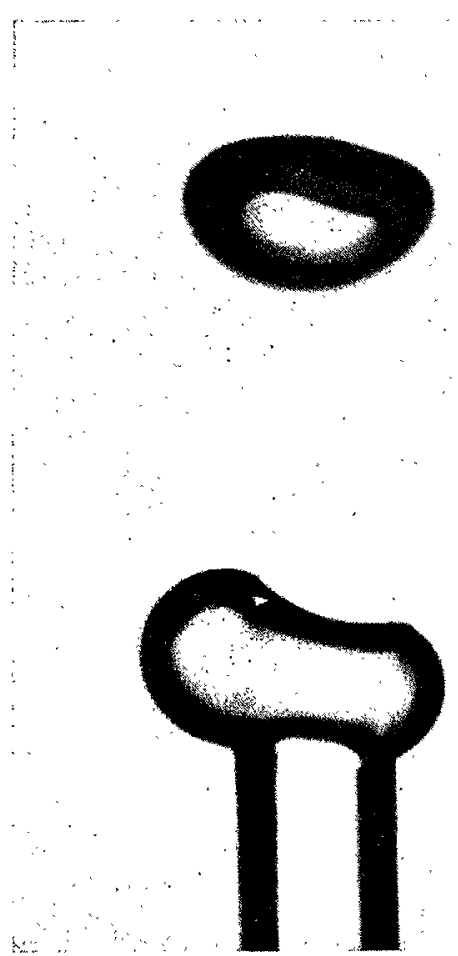

Pнот. 2
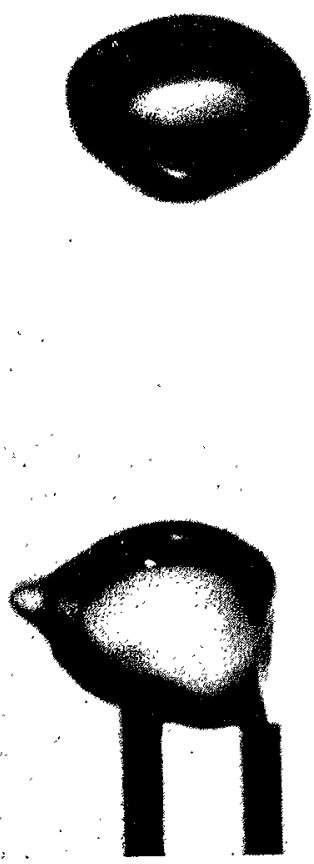

Pнот, 4

Evolution de la coalescence de deux bulles suivie grâce au retardateur électronique. Retards variant de 0 à 20 millisecondes.

Fréquence commune d'émission des bulles : 32 par seconde. Diamètre des injecteurs : $0,4 \mathrm{~mm}$. 
D IS C US S I O N

président : M. Chapouthien

M. le Président remercie M. SÉMÉnia et M. BêHar et s'assure auprès de M. BÉHaR que les mouvements de fluide chaufié que l'on voit sont dus uniquement à des rayons vagabonds.

M. BÉhar, sur la demande de M. le Président, donne les précisions suivantes:

Les rayons qui n'ont pas été déviés par les zones d'eau chaude restent parallèles entre eux et, de ce fait, passent par le foyer du deuxième objectif où ils seront occultés; ils ne peuvent donc atteindre directement l'écran, à l'exception, toutefois, de certains rayons diffractés par le bord du couteau.

La mise en évidence des rayons perturbés qui donnent une image des zones d'eau chaude, est surtout une question de contraste. Ces rayons perturbés apparaissent d'autant mieux que les rayons normaux, eux, sont masqués. Toutefois, en pratique, un arrêt trop complet des rayons normaux altérerait la qualité des images par suite de la diffraction. C'est pourquoi à qualité d'image et à contraste égaux, la sensibilité du montage, c'est-à-dire son aptitude à déceler de faibles déviations, est d'autant plus grande que la longueur focale du deuxième objectif est plus grande. Mais il est bien évident que, si on enlevait complètement le couteau, les rayons déviés ne donneraient que des taches claires (ou des zones sombres) imperceptibles sur le fond lumineux constitué par les rayons non déviés.

M. le Président remarque qu'en effet, l'image est d'une netteté surprenante et ajoute qu'au cours de son exposé, M. SÉméria s'est demandé si les spécialistes de la cavitation ne s'étaient pas préoccupés de savoir ce qui se passe quand la bulle s'en va, car c'est à ce moment-là qu'apparaît le phénomène thermique.

$N^{\prime} y$ a-t-il pas eu d'expériences analogues faites en hydraulique classique, où l'on s'est surtout préoccupé du moment où la bulle apparait?

M. Brymer indique que le collapse de bulles de cavitation a été observé de nombreuses fois à l'ultra-cinéma et plus rarement par la méthode Schlieren. Par ce dernier procédé, on a pu mettre en évidence, dans certains cas, l'existence d'ondes de choc ${ }^{\star}{ }^{*}$ ).

A propos d'applications hydrauliques des procédés strioscopiques, M. WrLlm fait remarquer qu'à l'inver'se de ce qu'ont recherché MM. BÉHAR et SÉmÉRI, il est possible de s'abstraire des phénomènes thermiques et visualiser exclusivement des gradients de pression : pour cela, il faut opérer aux environs de $4^{\circ}$ (pour l'eau) pour que les variations d'indice en fonction de la température soient faibles ou nuls, et observer avec un montage sensible, par exemple un strioscope en $Z$ à miroir avec ou sans contraste de phase. Dans ces conditions, on peut, pour fixer les idées, mettre en évidence facilement des gradients de pression de l'ordre de $1 \mathrm{bar} / \mathrm{cm}$ sous une épaisseur de $1 \mathrm{~cm}$, entrainant une déviation de l'ordre de $3 \mathrm{~s}$ (des déviations de l'ordre du dixième de seconde sont décelables).

A fortiori, les ondes de compression résultant du collapse de bulles de cavitation doivent être très visibles et même justiciables de procédés plus rudimentaires.

M. SÉmÉria précise qu'il faut une très haute sensibilité. De plus, la difficulté réside dans l'isolement du phénomène parmi les autres phénomènes parasites.

(*) Voir, par exemple, IR. Jones et D.H. EDwards : An experimental study of the forces gencrated by the collapse of transient cavities in water. Jourral of Fluid Mechanics, vol. 7 , $4^{\mathrm{e}}$ partie (avril 1960).

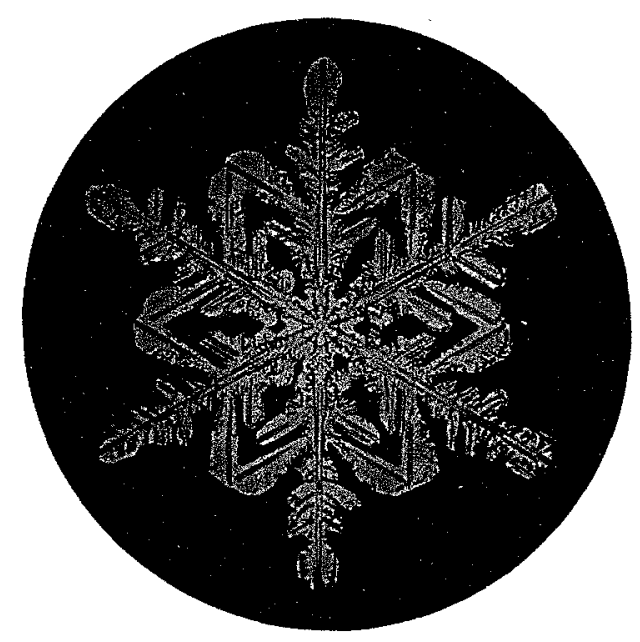

